\title{
Development of levofloxacin-loaded PLGA microspheres of suitable properties for sustained pulmonary release
}

\author{
Marisa C. Gaspar ${ }^{\mathrm{a}, \mathrm{b}, \mathrm{c}, *}$, Alberto A.C.C. Pais ${ }^{c}$, João J.S. Sousa ${ }^{\mathrm{b}}$, Julien Brillaut ${ }^{\mathrm{d}, \mathrm{e}}$, \\ Jean-Christophe Olivier ${ }^{\mathrm{d}, \mathrm{e}}$ \\ ${ }^{a}$ Chemical Process Engineering and Forest Products Research Centre (CIEPQPF), Department of Chemical Engineering, University of Coimbra, Rua Sílvio Lima, Pólo II, \\ Pinhal de Marrocos, 3030-790 Coimbra, Portugal \\ ${ }^{\mathrm{b}}$ Laboratory of Pharmaceutical Technology, Faculty of Pharmacy, University of Coimbra, Pólo das Ciências da Saúde, Azinhaga de Santa Comba, 3000-548 Coimbra, \\ Portugal \\ ${ }^{\mathrm{c}}$ Department of Chemistry, University of Coimbra, 3004-535 Coimbra, Portugal \\ ${ }^{\mathrm{d}}$ INSERM, U 1070, Pôle Biologie Santé, 1 rue Georges Bonnet, TSA 51106, 86073 Poitiers, Cedex 9, France \\ ${ }^{\mathrm{e}}$ University of Poitiers, Faculty of Medicine and Pharmacy, 6 rue de la Milétrie, TSA 51115, 86073 Poitiers, Cedex 9, France
}

\section{A R T I C L E I N F O}

\section{Keywords:}

Cystic fibrosis

Lung delivery

Levofloxacin-loaded PLGA MS

Physico-chemical characterization

Controlled release

In vitro studies

\begin{abstract}
A B S T R A C T
Aerosol antibiotics are an interesting alternative to oral or intravenous therapy in Cystic Fibrosis lung infections. Levofloxacin (LVX) inhaled solution is already an effective option. In this study, the aim was the development of LVX-loaded PLGA microspheres (MS) for pulmonary administration as a dry powder. MS were prepared, for the first time, by a modified double emulsion solvent evaporation method with premix membrane homogenization. Aqueous phases were saturated with LVX and a fatty acid (lauric acid) was added to avoid the drug escaping from the organic phase. MS were characterized in terms of size, drug content, morphology and in vitro release properties. X-ray diffraction, Fourier-transform infrared spectroscopy, differential and gravimetric thermal analysis, and cytotoxicity analyses were performed. Results showed this new method increased the drug loading while maintaining an adequate $(\sim 5 \mu \mathrm{m})$ particle size and controlled release. Compared to a solution for inhalation, these properties combined with the dry-powder nature of these MS will improve patient compliance. The incorporation of lauric acid was not advantageous because the particle size was higher and no improvements concerning the sustained release occurred. LVX was molecularly dispersed in the matrix, or it was in amorphous state, as confirmed by the physico-chemical analyses. Calu- 3 cell viability assays demonstrated no cytotoxicity for these MS, making them a promising system for LVX pulmonary delivery.
\end{abstract}

\section{Introduction}

The delivery of antibiotics at the site of infection has been demonstrated to be efficient in the treatment of lung infections (Giron Moreno et al., 2011). In the Cystic Fibrosis (CF) lung disease, the development of chronic infection by Pseudomonas aeruginosa is the predominant cause of the morbidity and mortality and its control is the main objective of long term antibiotherapy (Geller, 2009; Giron Moreno et al., 2011; Bjarnsholt et al., 2009). Several nebulized formulations of antibiotics have been developed and marketed recently, which allow high local concentrations and a reduced systemic toxicity risk (Gaspar et al., 2013; Tiddens et al., 2014). The nebulized solution of levofloxacin (LVX, Fig. 1) (Quinsair ${ }^{\circledR}$ in Europe or Aeroquin ${ }^{\mathrm{TM}}$ in EUA) was demonstrated to be effective in improving the lung function of $\mathrm{CF}$ patients
(EMA, 2015; Foundation, 2015). However, the present solutions for inhalation require time-consuming hygienic procedures for the administration devices and long administration times, several times a day, that preclude optimal observance of the treatment (Heijerman et al., 2009). Thus, the formulation of LVX as readily administrable dry powder for inhalation is an interesting option to improve drug product stability, CF patients' comfort and treatment efficiency. In addition, the use of sustained-release biodegradable and biocompatible polymerbased microspheres (MS), administered in a dry powder inhaler, may further improve patients' comfort by reducing the frequency of administration. The hydrophobic copolymer poly (DL-lactide-co-glycolide) (PLGA) is of particular relevance for the preparation of sustained release drug delivery systems, since it is biodegradable and biocompatible. The preparation methods of PLGA-based carriers for

\footnotetext{
* Corresponding author at: CIEPQPF, Department of Chemical Engineering, University of Coimbra, Rua Sílvio Lima, Pólo II, Pinhal de Marrocos, 3030-790 Coimbra, Portugal.

E-mail address: marisagaspar@eq.uc.pt (M.C. Gaspar).
} 
<smiles>C[C@H]1COc2c(N3CCN(C)CC3)c(F)cc3c(=O)c(C(=O)O)cn1c23</smiles>

Fig. 1. Chemical structure of LVX.

hydrophilic drugs include the double $\mathrm{W}_{1} / \mathrm{O} / \mathrm{W}_{2}$ emulsion-solvent evaporation process, the $\mathrm{W} / \mathrm{O}$ phase separation, and the Hydrophobic Ion Pairing technique. Both $\mathrm{W} / \mathrm{O}$ phase separation and Hydrophobic Ion Pairing were shown to be efficient methods for entrapping hydrophilic drugs within PLGA. The former is however complex to set up and necessitates a large amount of organic solvent and non biodegradable oil, such paraffin or silicon oils (Sah and Sah, 2015). The latter requires to select an appropriate, non-toxic counter ion, which, in the case of positively-charged drug may be a fatty acid (Govender et al., 1999). The $\mathrm{W}_{1} / \mathrm{O} / \mathrm{W}_{2}$ solvent evaporation method employs low amounts of organic solvent and has been extensively used and studied. The main issue of the $\mathrm{W}_{1} / \mathrm{O} / \mathrm{W}_{2}$ solvent evaporation method however is the difficulty to control the migration of the water soluble drug from the inner to the outer aqueous phase, which generally determines low drug contents (Govender et al., 1999). Incorporating the water soluble LVX drug (FDA, 2006) within PLGA MS and promoting a high entrapment efficiency is therefore a challenging issue (FDA, 2006). The aim of this work was to prepare inhalable LVX-loaded PLGA MS using, for the first time, a modified double emulsion solvent evaporation method for the controlled release of LVX in the lungs. Compared to a solution for inhalation, the dry-powder nature of such MS is expected to improve patient compliance, due to an easier administration procedure and to the lower frequency of administration. The premix membrane homogenization technique was used in order to obtain narrowly size-distributed MS (Doan and Olivier, 2009).

\section{Materials and methods}

\subsection{Materials}

Resomer $^{\circledast}$ RG 502H (PLGA 50:50, MW: 7000-17000, acid terminated), Resomer ${ }^{\circledR}$ RG 503 (PLGA 50:50, MW: 24,000-38,000, ethyl ester terminated), lauric acid, dimethyl sulfoxide (DMSO), Dulbecco's modified Eagle's medium (DMEM) nutrient mixture F-12 and fetal bovine serum (FBS) were obtained from Sigma-Aldrich ${ }^{\circledR}$ (St. Quentin Fallavier, France). Rhodoviol 4/125 (polyvinylalcohol (PVA)), degree of hydrolysis of $88 \%$ was purchased from Prolabo (Paris, France).
Levofloxacin hemihydrate was kindly provided by Tecnimede S.A. (Lisbon, Portugal). Dichloromethane (DCM) HipPerSolv ${ }^{\mathrm{TM}}$ Chromanorm for HPLC was purchased from $\mathrm{BDH}^{\circledR}$ (VWR analytical, Fontenay-sousBois, France). Formic acid 99-100\% AnalaR (NormaPur) was obtained from $\mathrm{VWR}^{\circledast}$ (Fontenay-sous-Bois, France) and acetonitrile of HPLC grade was purchased from Carlo Erba reagents (Val de Reuil, France). 3(4,5-dimethylthiazol-2-yl)-5-(3-carboxymethoxyphenyl)-2-(4-sulfophenyl)-2 tetrazolium (MTS) CellTiter $96^{\circledR}$ AQueous One Solution Cell Proliferation Assay was purchased from Promega (Charbonnières-lesBains, France). Hanks' Balanced Salt Solution (HBSS) was obtained from GIBCO $^{\circledR}$ Thermo Fisher Scientific ${ }^{\mathrm{TM}}$ (Illkirch, France). All other chemicals were of analytical grade or equivalent. Purified water was produced using a MilliQ Gradient ${ }^{\circledR}$ Plus Millipore system.

\subsection{PLGA MS preparation}

Briefly, LVX-loaded PLGA MS were prepared by a double emulsion solvent evaporation method with a premix membrane homogenization step (Doan et al., 2011; Doan and Olivier, 2009). In order to select the polymer grade, the experimental conditions were the following (Formulations 1 and 2, Table 1). A volume of $0.6 \mathrm{~mL}$ of a LVX solution (water phase $1, \mathrm{~W}_{1}$, adjusted to $\mathrm{pH} 6$ with hydrochloric acid) was emulsified into $3 \mathrm{~mL}$ of a PLGA solution in DCM (oil phase O) using a Polytron ${ }^{\circledR}$ PT $3100 \mathrm{D}$ homogenizer equipped with a $7 \mathrm{~mm}$ homogenizing accessory (Kinematica AG, Switzerland) and set at 30,000 rpm for $30 \mathrm{~s}$. The obtained $\mathrm{W}_{1} / \mathrm{O}$ emulsion was dispersed in $7 \mathrm{~mL}$ of a $3 \%(\mathrm{w} / \mathrm{v})$ PVA solution in PBS at $\mathrm{pH} 7.4$ (water phase $2, \mathrm{~W}_{2}$ ) under magnetic stirring (400 rpm). The resulting $\mathrm{W}_{1} / \mathrm{O} / \mathrm{W}_{2}$ emulsion was subjected to three homogenization cycles through a Shirasu porous glass (SPG) membrane (19.9 $\mu \mathrm{m}$ porosity) under $25 \mathrm{kPa}$ transmembrane pressure using an external pressure-type micro kit emulsification device (SPG Technology, Sadowara, Japan). It was immediately poured into $25 \mathrm{~mL}$ of a $0.4 \%$ (w/ v) PVA solution in PBS (Water phase $3, \mathrm{~W}_{3}$ ) under magnetic stirring (400 rpm). DCM was evaporated under low pressure at room temperature during $10 \mathrm{~min}$ using a rotatory evaporator. MS were then washed through three cycles of centrifugation ( $3500 \mathrm{rpm}, 5 \mathrm{~min}$ ) and re-suspended in purified water and finally freeze-dried. In a second step, the effect of the addition of LVX to the O phase and to the water phases $\mathrm{W}_{2}$ and $\mathrm{W}_{3}$ (Formulations 3-4, Table 1) was investigated, as well as the addition of lauric acid in the $\mathrm{O}$ phase (Formulation 5 , Table 1 ), in the LVX-to-lauric acid molar ratio of $1: 1$, as previously described (Govender et al., 1999).

\subsection{Characterization of LVX-loaded PLGA MS}

\subsubsection{Particle size analysis}

The mean size of the volume distribution $\left(D_{v}\right)$ was evaluated by dispersing the MS in purified water using laser light diffraction (Microtrac ${ }^{\circledR}$ X100 particle size analyzer) (Gaspar et al., 2015).

Table 1

Details of formulation, including the amount of polymer and drug used for the MS preparation in the different phases.

\begin{tabular}{|c|c|c|c|c|c|c|c|c|c|}
\hline \multirow[t]{2}{*}{ Formulation number } & \multirow[t]{2}{*}{$\begin{array}{l}\text { PLGA } \\
\text { Grade }\end{array}$} & \multirow{2}{*}{$\begin{array}{l}\text { Water phase } \mathrm{W}_{1} \\
(0.6 \mathrm{~mL} \text { water }) \\
\mathrm{LVX} \\
(\mathrm{mg})\end{array}$} & \multicolumn{3}{|c|}{$\begin{array}{l}\text { Oil phase } \mathrm{O} \\
(3 \mathrm{~mL} \mathrm{DCM})\end{array}$} & \multicolumn{2}{|c|}{$\begin{array}{l}\text { Water phase } \mathrm{W}_{2} \\
(7 \mathrm{~mL} \text { PBS, pH 7.4) }\end{array}$} & \multicolumn{2}{|c|}{$\begin{array}{l}\text { Water phase } \mathrm{W}_{3} \\
(25 \mathrm{~mL} \text { PBS, pH 7.4) }\end{array}$} \\
\hline & & & $\begin{array}{l}\text { PLGA } \\
(\mathrm{mg})\end{array}$ & $\begin{array}{l}\text { LVX } \\
\text { (mg) }\end{array}$ & $\begin{array}{l}\text { Lauric acid } \\
\text { (mg) }\end{array}$ & $\begin{array}{l}\text { PVA } \\
(\%, \mathrm{w} / \mathrm{v})\end{array}$ & $\begin{array}{l}\text { LVX } \\
(\%, w / v)\end{array}$ & $\begin{array}{l}\text { PVA } \\
(\%, w / v)\end{array}$ & $\begin{array}{l}\text { LVX } \\
(\%, w / v)\end{array}$ \\
\hline 1 & RG $502 \mathrm{H}$ & 150 & 300 & - & - & 3 & - & 0.4 & - \\
\hline 2 & RG 503 & 150 & 300 & - & & 3 & - & 0.4 & - \\
\hline 3 & RG $502 \mathrm{H}$ & 150 & 300 & 100 & - & 3 & 3.5 & 0.4 & 3.2 \\
\hline 4 & RG 502H & 150 & 300 & 200 & - & 3 & 3.5 & 0.4 & 3.2 \\
\hline 5 & RG 502H & 150 & 300 & 100 & 54 & 3 & 3.5 & 0.4 & 3.2 \\
\hline
\end{tabular}

\footnotetext{
* Lauric acid was added in the LVX-to-lauric acid molar ratio of 1:1.
} 


\subsubsection{Drug content and entrapment efficiency}

The amount of LVX in the MS was quantified by spectrophotometry at $300 \mathrm{~nm}$ using a Varian Cary $50 \mathrm{UV}-$ Visible spectrophotometer after MS dissolution in DMSO (LVX calibration curve 0.625-10 $\mu \mathrm{g} / \mathrm{mL}$ ). Drug contents (\%) were calculated as the percentage of the amount (mg) of LVX per total amount of MS (mg). Entrapment efficiency (\%) was calculated as the percent ratio of the actual (experimentally determined) LVX content to the theoretical considering a $100 \%$ entrapment efficiency.

\subsubsection{In vitro drug release studies}

The LVX in vitro release study was performed under sink conditions by dispersing $5 \mathrm{mg}$ of LVX-loaded MS in $10 \mathrm{~mL}$ of PBS, $\mathrm{pH}=7.4$, under magnetic stirring $(600 \mathrm{rpm})$ at $37^{\circ} \mathrm{C}$. For the LVX determination by HPLC, aliquots of $1 \mathrm{~mL}$ were collected at pre-determined points and subjected to centrifugation for $5 \mathrm{~min}$ at $3500 \mathrm{rpm}$ (Hettich ${ }^{\circledR}$ Zentrifugen Universal 320R, Germany). Then, $100 \mu \mathrm{L}$ of the supernatant were collected for LVX determination by HPLC. The remaining volume was vortex-mixed and added back to the flasks (Gaspar et al., 2015).

\subsubsection{Scanning electron microscopy (SEM)}

Morphology and surface appearance of MS were analyzed by SEM after gold-sputtering the freeze-dried MS in argon atmosphere. It was performed using a Jeol JSM 6010 LV electron microscope (Tokyo, Japan) at $15 \mathrm{kV}$.

\subsection{Additional characterization of the selected PLGA formulation}

\subsubsection{Powder X-ray diffraction (XRD)}

For XRD analysis, samples were placed in a low background silicon holder in Bragg-Brentano configuration, with a copper tube powered at $45 \mathrm{kV}$ and $40 \mathrm{~mA}$. The scanning was made in the range $5^{\circ}<2 \theta<45^{\circ}$ at a step of $0.066^{\circ}$ and time/step of $10 \mathrm{~s}$ in an Empyrean PANalytical (The Netherlands) diffractometer with the detector Xcelerator in scanning mode and opened at $2^{\circ}$. The $\mathrm{K} \beta$ component was eliminated by using a nickel filter in a secondary optic.

\subsubsection{Differential thermal Analysis/ thermal gravimetric analysis (DTA/ TGA)}

A SDT Q600 Instrument (TA, USA) was operated from $30^{\circ} \mathrm{C}$ to $300^{\circ} \mathrm{C}$ with a $10^{\circ} \mathrm{C} / \mathrm{min}$ heating rate and under a $100 \mathrm{~mL} / \mathrm{min}$ air flow rate. An aluminum empty pan was used as a reference and calibration was carried out with a sapphire.

\subsubsection{Attenuated total reflectance infrared spectroscopy (ATR-FTIR)}

A FT-IR 6700 spectrometer (Thermo Scientific Nicolet ${ }^{\mathrm{TM}}$, USA) equipped with an ATR accessory was used to perform the IR analysis with 16 scans between 4000 and $650 \mathrm{~cm}^{-1}$ for each spectrum $\left(4 \mathrm{~cm}^{-1}\right.$ resolution).

\subsubsection{Aerodynamic properties}

The mass median aerodynamic diameter (MMAD), the emitted dose (ED, \%), and the fine particle fraction (FPF, \%) were evaluated using a Next Generation Impactor (NGI, Copley Ltd., Nottingham, UK). A sizethree transparent gelatin capsule was filled with $20 \pm 1 \mathrm{mg}$ of MS powder, inserted in a dry-powder inhaler Handihaler ${ }^{\circledR}$ (BoehringerIngelheim, Germany) and pierced. The pump was turned on for $4 \mathrm{~s}$ at a $60 \mathrm{~L} / \mathrm{min}$ of flow rate (Gaspar et al., 2015). This flow rate was applied in order to obtain a high pressure drop in the dry-powder inhaler in order to facilitate the powder dispersion. The powder fractions remaining in the inhaler and the capsule or deposited on the induction port and on all the stages of the NGI were collected using DMSO as the solvent. Then, LVX was quantified by spectrophotometry (see Section 2.3.2).

\subsubsection{Cross-section analysis}

In order to understand the internal structure of MS, they were incorporated in a resin (at $35^{\circ} \mathrm{C}$, for $24 \mathrm{~h}$, avoiding the MS melting) and cross-sections were after obtained with the Microtome Leica EM UC6 (Leica Co, Austria), before observation. For SEM analyses, MS were incorporated in Spurr's resin (Spurr low-viscosity embedding kit, Sigma-Aldrich ${ }^{\oplus}$, Germany), and cross-sections of $1.5 \mu \mathrm{m}$ of thickness then observed (SEM, Jeol ISM 5600 LV microscope), after platinumsputtering the samples in argon atmosphere. In the case of TEM, incorporation in epoxy resin (epoxy embedding resin kit, Fluka analytical, Sigma-Aldrich ${ }^{\oplus}$, Germany) was carried and $80 \mathrm{~nm}$ cross-sections were then stained with aqueous lead citrate solution $(0.2 \%(\mathrm{w} / \mathrm{v}), 10 \mathrm{~min})$ before observation (TEM, FEI-Tecnai ${ }^{\mathrm{TM}}$ G2 Spirit BioTwin; AnalySIS 3.2 acquisition software).

\subsubsection{Specific surface area}

The specific surface area was determined by the $\mathrm{N}_{2}$ adsorption BET multipoint method, with 6 points in the relative pressure range of 0.1-0.3, using a Micrometrics ASAP 2020 v 2.04 (Tewes et al., 2013). Samples were purged overnight under $\mathrm{N}_{2}$ at room temperature.

\subsubsection{MTS assay}

The in vitro cytotoxicity of PLGA MS on Calu- 3 cell line was evaluated with the MTS assay. Calu-3 cells were obtained from the American Type Culture Collection (Rockville, MD). The cells were grown in DMEM/F-12 nutrient mixture supplemented with L-glutamine $(2 \mathrm{mM})$ and FBS $\left(10 \%(\mathrm{v} / \mathrm{v})\right.$ in $90-95 \%$ relative humidity with $5 \% \mathrm{CO}_{2} /$ $95 \%$ atmospheric air at $37^{\circ} \mathrm{C}$. For the MTS assay, the cells were seeded at a density of $1 \times 10^{4}$ cells/well in 96-well plates and grown in the same conditions for $24 \mathrm{~h}$. Just before the assay, LVX-loaded PLGA MS, unloaded MS or the LVX powder were mixed with the medium for cell culture to obtain solutions/suspensions with a LVX concentration of $2 \mathrm{mg} / \mathrm{mL}$ and a PLGA concentration close to $20 \mathrm{mg} / \mathrm{mL}$ (Osman et al., 2013). In the 96-well plates, the medium was aspirated and replaced with $100 \mu \mathrm{L}$ of pre-warmed culture medium. Then, $100 \mu \mathrm{L}$ of LVX solution/MS suspensions were added to obtain the highest LVX concentration tested $(1 \mathrm{mg} / \mathrm{mL})$. Serial dilutions were conducted to obtain a LVX concentration range in the $0.0156-1 \mathrm{mg} / \mathrm{mL}$ range (Osman et al., 2013). For PLGA MS, the concentration ranged between 0.156 and $10 \mathrm{mg} / \mathrm{mL}$. Cells without treatment were used for $100 \%$ viability control. After $24 \mathrm{~h}$ of incubation, the medium was replaced by $120 \mu \mathrm{L}$ of pre-warmed MTS reagent diluted in HBSS $(20 \%(\mathrm{v} / \mathrm{v}))$, allowing the reduction of the tetrazolium compound by cells into a soluble formazan product. The absorbance was measured by spectrophotometry after $2 \mathrm{~h}$, at $490 \mathrm{~nm}$ using a microplate reader $\left(\right.$ Varioskan $^{\mathrm{TM}}$, Thermo Scientific ${ }^{\mathrm{TM}}$, USA). A second measurement at $650 \mathrm{~nm}$ was done to subtract the background (Adi et al., 2010). Experiments were done in five different occasions. The cell viability (\%) was calculated as follows in Eq. (3),

Cell viability $(\%)=\frac{\mathrm{A}_{490 \mathrm{~nm}^{-}} \mathrm{A}_{650 \mathrm{~nm}}}{\mathrm{~A}_{\text {control } 490 \mathrm{~nm}}-\mathrm{A}_{\text {control 650nm }}} \times 100$

where $A_{490 n m}$ corresponds to the absorbance from the wells incubated with LVX and PLGA MS at $490 \mathrm{~nm}, \mathrm{~A}_{650 \mathrm{~nm}}$ to the respective background absorbance at $650 \mathrm{~nm}$ and $A_{\text {control } 490 \mathrm{~nm}}$ and $A_{\text {control } 650 \mathrm{~nm}}$ to the absorbance of the control wells.

Cell viability (\%) versus log concentration curves were fitted for each experiment using Equation (4) with GraphPad software (GraphPad Prism version 5.00 for Windows, San Diego California USA),

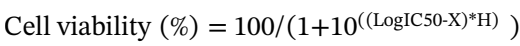

where IC50 is the concentration that inhibits $50 \%$ of cell growth, i.e. cell viability decreased in 50\% (Anderson and Shive, 2012), X the logarithmic concentration of the formulation and $\mathrm{H}$ the Hillslope number. 
Table 2

Particle size, drug content and encapsulation efficiency of PLGA MS. Results are expressed as mean $\pm \operatorname{SD}(\mathrm{n}=3)$.

\begin{tabular}{lllll}
\hline $\begin{array}{l}\text { Formulation } \\
\text { number }\end{array}$ & PLGA type & $D_{v}(\mu \mathrm{m})^{\mathrm{a}}$ & $\begin{array}{l}\text { LVX content } \\
\text { (wt.\%) }\end{array}$ & $\begin{array}{l}\text { Entrapment } \\
\text { efficiency (\%) }\end{array}$ \\
\hline $1^{\mathrm{b}}$ & RG 502H & 4.6 & 3.30 & 9.91 \\
$2^{\mathrm{b}}$ & RG 503 & 4.3 & 0.30 & 0.90 \\
3 & RG 502H & $5.0 \pm 1.7^{\mathrm{d}}$ & $10.5 \pm 1.4^{\mathrm{d}}$ & $23.1^{\mathrm{c}}$ \\
4 & RG 502H & $4.5 \pm 0.1$ & $9.3 \pm 0.7$ & $17.3^{\mathrm{c}}$ \\
5 & RG 502H & $17.5 \pm 1.0$ & $18.4 \pm 0.2$ & $44.4^{\mathrm{c}}$ \\
\hline
\end{tabular}

a Laser light diffraction method.

b $\mathrm{n}=1$.

c For the calculation of the entrapment efficiency, the LVX fractions that were dissolved in the outer water phases $\mathrm{W}_{2}$ and $\mathrm{W}_{3}$ were not considered.

d Data published in (Gaspar et al., 2016).

\subsection{Statistical analysis}

All analyses were carried out with the GraphPad software. Statistical analysis of cell viability was performed from IC50 values ( $n=5$ ) by one-way ANOVA followed by post hoc Dunnett's multiple comparison test. Statistical significance was considered with a $p$ value $<0.05$

\section{Results and discussion}

\subsection{Microsphere formulation selection}

LVX-loaded PLGA MS were prepared by the $\mathrm{W}_{1} / \mathrm{O} / \mathrm{W}_{2}$ solvent evaporation method based on previously described procedures (Doan et al., 2011; Doan and Olivier, 2009). In a first step, two PLGA polymers of different grades, the acid terminated Resomer ${ }^{\circledR} \mathrm{RG} 502 \mathrm{H}$ and the ethyl ester end-capped Resomer ${ }^{\circledast}$ RG 503 were investigated and their impact on the microsphere size and LVX content was evaluated as the first selection criteria (Table 2). For efficient deposition in the lungs, aerosols for inhalation should be made of particles with aerodynamic diameters between 1 and $5 \mu \mathrm{m}$ (Emami et al., 2009; Høiby, 2011). The target value for the $D_{v}$ values of the PLGA microsphere ranged therefore from 2 to $5 \mu \mathrm{m}$. In the particular case of antibiotics like LVX, which need a relatively high drug dose for therapeutic efficiency, the MS drug content, i.e. the drug-to-excipient ratio, is of utmost importance to minimize toxicity risks arising from the excipient materials delivered into the patients' lungs. Our minimal target value for the LVX content was set at $10 \%(\mathrm{w} / \mathrm{w})$.

The mean diameter values $D_{v}$ were satisfactory for lung delivery with formulations 1 and 2, but the LVX content values were below the target. This can be ascribed to the significant water solubility of LVX in PBS, pH 7.4 (50 mg/mL), (FDA, 2006), and to the high $\left(\mathrm{W}_{2}+\mathrm{W}_{3}\right.$ )-to$\left(\mathrm{W}_{1} / \mathrm{O}\right)$ volume ratio, giving LVX a preferential distribution in the outer aqueous phase at the expense of the inner $\mathrm{W}_{1}$ phase and $\mathrm{O}$ phase, as already reported for water soluble drugs (Govender et al., 1999). MS prepared with PLGA RG 502H presented the highest LVX content in agreement with results previously reported (Kumar et al., 2012). Due to the free carboxylic end groups, the low molecular weight PLGA RG $502 \mathrm{H}$ polymer was more hydrophilic than the higher molecular weight end-capped PLGA RG 503 polymer, and established better interactions with LVX (Kumar et al., 2012; Lecaroz et al., 2006). Ion pairing between the carboxylate groups of the PLGA polymer and the zwitterionic LVX may also favor LVX entrapment. The RG 502H polymer grade was therefore chosen for further investigations.

In the case of formulations 3 and 4 , the saturation of the $W_{2}$ and $W_{3}$ phases with LVX substantially increased the LVX content compared to formulation 1 (Table 1), showing that minimizing the effect of the diffusion gradient was an efficient means to increase drug loading. However, doubling the LVX concentration in the oil phase $\mathrm{O}$ had no impact upon LVX content. Formulations 3 and 4 resulted in MS with $D_{v}$ values close to that obtained with formulation 2 and, therefore, within the target values. In agreement to previous work (Govender et al., 1999), a higher LVX content was obtained when lauric acid was added to the oil phase $\mathrm{O}$ (Formulation 5), but the diameter $D_{v}$ was largely above the target. The higher content of LVX obtained with the addition of lauric acid was attributed to the formation of lipophilic ion pairs between LVX and lauric acid through electrostatic interactions between the acidic moiety of lauric acid and the basic piperazinyl group of LVX, decreasing the diffusion of the drug into the aqueous outer phase.

SEM images of the MS prepared with formulations 3 and 4 (Fig. 2A and $2 \mathrm{~B}$ ) revealed spherical shape, with an apparent diameter in agreement with the determined mean diameter $D v$. The surface was smooth, with no visible pores. Some small "specks" are present and were attributed to residual PVA. In the case of Formulation 5 (Fig. 2C), the MS were apparently of the same size as in MS of formulations 3 and 4 , but they showed a tendency to aggregation which may explain the larger $D_{v}$ determined by laser light diffraction method in water (Table 2). Some of the MS are distorted, with wrinkled surfaces, and also contain "specks" of various sizes.

For the PLGA MS prepared according to formulations 3 and 4 (Fig. 3), very similar release profiles were obtained in sink conditions. They were characterized by a "burst" effect from 35 to $40 \%$ within the first $30 \mathrm{~min}$ followed by a slow release up to a maximum of $75 \%$ at $72 \mathrm{~h}$. For the MS obtained with formulation 5, i.e. with the lauric acid additive, the "burst" release clearly became dominant, with $70 \%$ of LVX released within $30 \mathrm{~min}$. These results should not be considered as fully predictive of the in vivo release profiles. Indeed, due to the low volume of epithelial lining fluid and to the likely phagocytosis of at least a fraction of the microspheres (with a $1-10 \mu \mathrm{m}$ diameter range) by the lung macrophages (Diab et al., 2012), release times were reported to be longer in vivo than in vitro (Doan et al., 2013). However, to date no standardized in vitro methods are available to predict in vivo release profiles after administration into lungs of sustained release formulations. Therefore, sink conditions is the only comparable way to characterize release profiles.

MS partial degradation was observed after one week in the release medium for all the formulations (Fig. 2A1, B1 and C1). Complete erosion may occur within 2 weeks, as already reported in the literature for RG 502H particles (Díez and Tros de Ilarduya, 2006). Therefore, the RG $502 \mathrm{H}$ PLGA polymer is expected to minimize pulmonary accumulation of polymer after MS lung deposition, especially in the case of repeated administrations.

Both MS obtained with formulations 3 and 4 had adequate and similar drug contents, particle sizes and sustained release profiles. But, due to the lower amount of LVX needed for their preparation, the MS formulation 3 was selected for further studies.

\subsection{Further analysis of the selected PLGA formulation}

Further characterization was done on the selected MS (Formulation 3).

\subsection{1. $X R D$}

The XRD spectra of LVX hemihydrate, PVA, PLGA RG 502H and MS (Formulation 3) are shown in Fig. 4. LVX hemihydrate presented sharp peaks at several diffraction angles $\left(6.5^{\circ}, 9.7^{\circ}, 13.1^{\circ}, 15.7^{\circ}, 19.3^{\circ}, 19.9^{\circ}\right.$ and $26.6^{\circ}$ ) denoting its crystalline nature, in agreement with previous work (Gorman et al., 2012; Hema et al., 2009; Kitaoka et al., 1995). Diffuse scattering and a broad small peak was observed at $19.3^{\circ}$ for PVA confirming its semi-crystalline nature (Guirguis and Moselhey, 2012). The commercial powder of the PLGA polymer appeared to be amorphous. In the case of the LVX-loaded PLGA MS, no diffraction peak was observed, indicating that LVX is either solubilized, or dispersed in an amorphous state or as nanocrystals in the PLGA polymer matrix. 

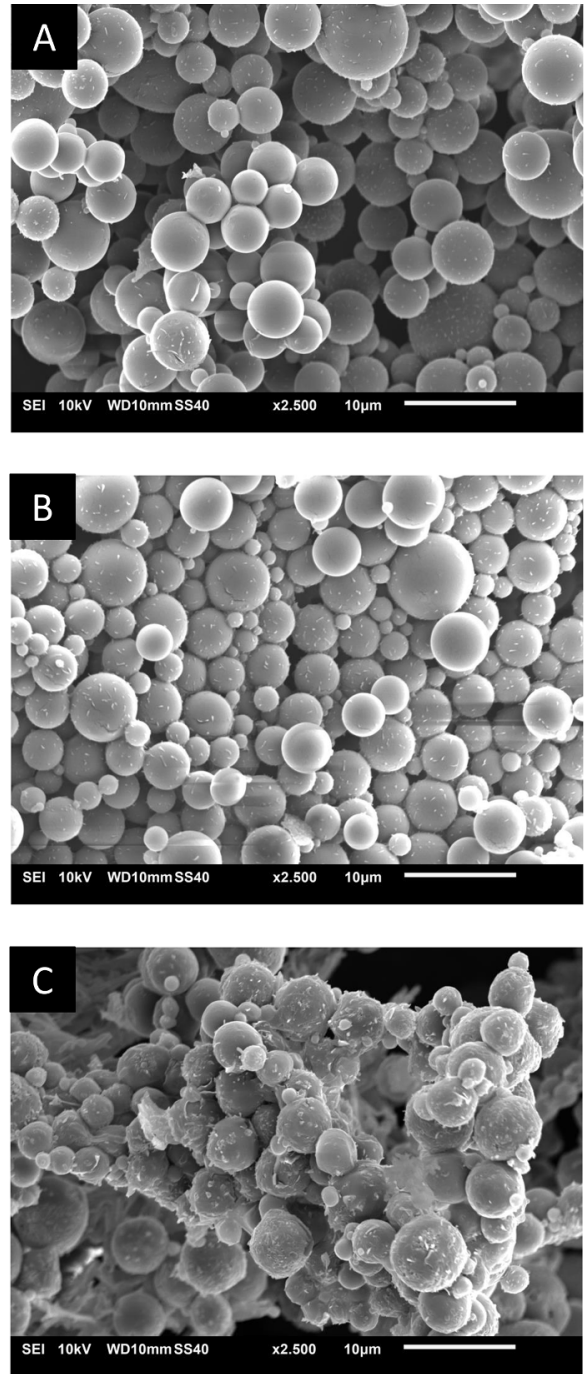
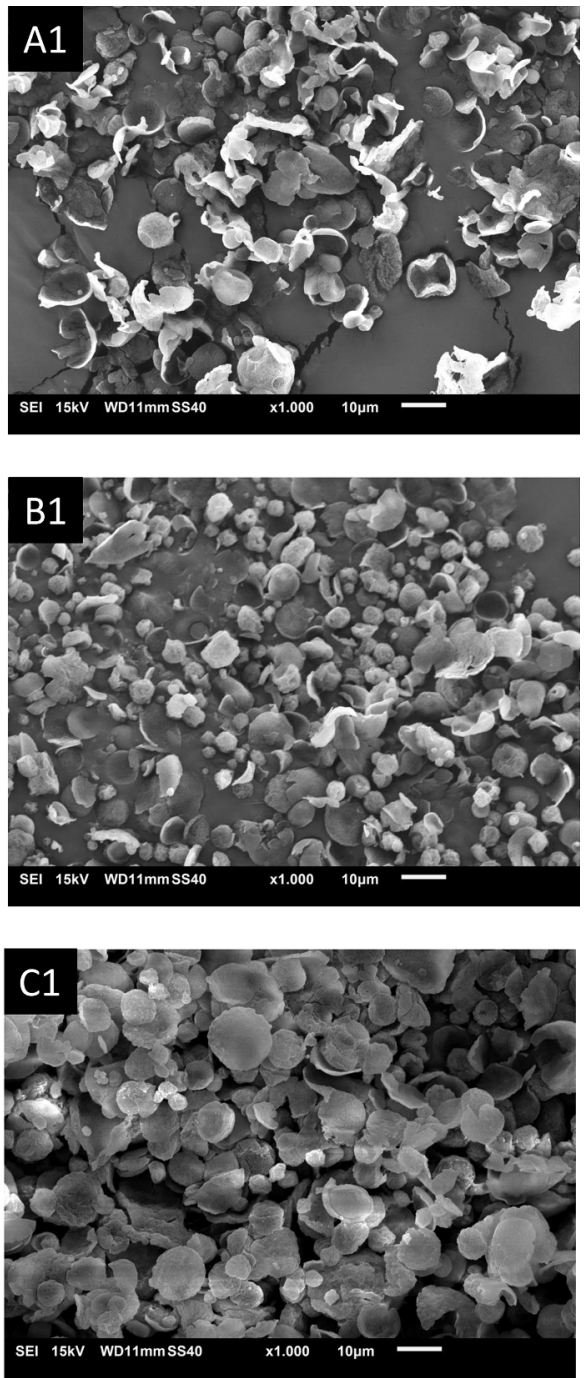

Fig. 2. SEM images of PLGA MS observed after preparation: (A) Formulation 3; (B) Formulation 4 and (C) Formulation 5 and after one week of incubation in PBS at $37^{\circ} \mathrm{C}$ and $\mathrm{pH} 7.4$ under magnetic stirring of $600 \mathrm{rpm}$, respectively: (A1); (B1) and (C1).

\subsubsection{DTA/TGA}

For LVX (hemihydrate commercial product) (Fig. 5A), two endothermic peaks were observed at $78{ }^{\circ} \mathrm{C}$ and $225^{\circ} \mathrm{C}$. The first endothermic transition over a broad temperature range (around $40-80^{\circ} \mathrm{C}$ )

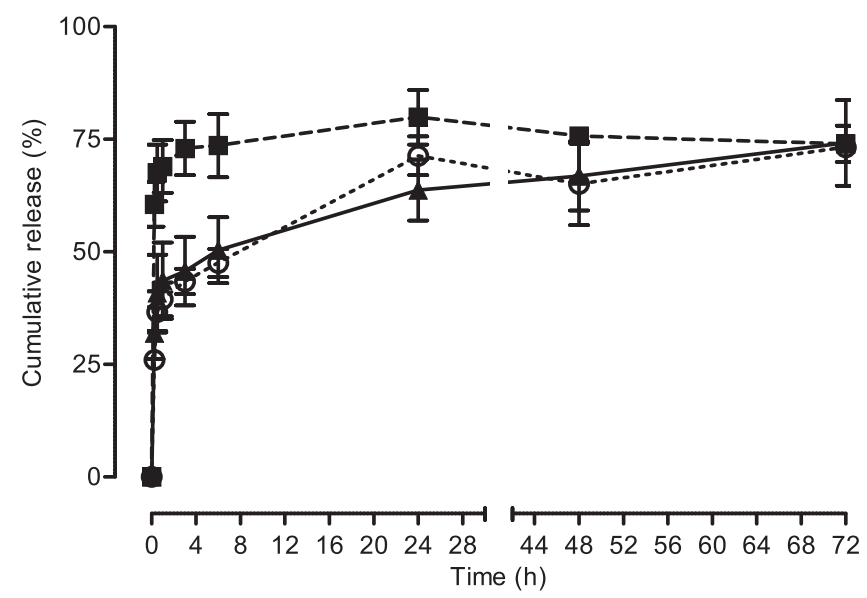

Fig. 3. LVX in vitro cumulative release profile from LVX-loaded PLGA MS in PBS, at $\mathrm{pH}=7.4$ and $37^{\circ} \mathrm{C}$ : Formulation 3(\lrcorner$)$; Formulation $4(-a)$; and Formulation 5 (-口-). Results are expressed as mean \pm SEM $(n=3)$. was attributed to the water loss of the hemihydrate and was accompanied with a weight loss of $2.35 \%$ in agreement with a previous report (Gorman et al., 2012; Kitaoka et al., 1995). The second endothermic

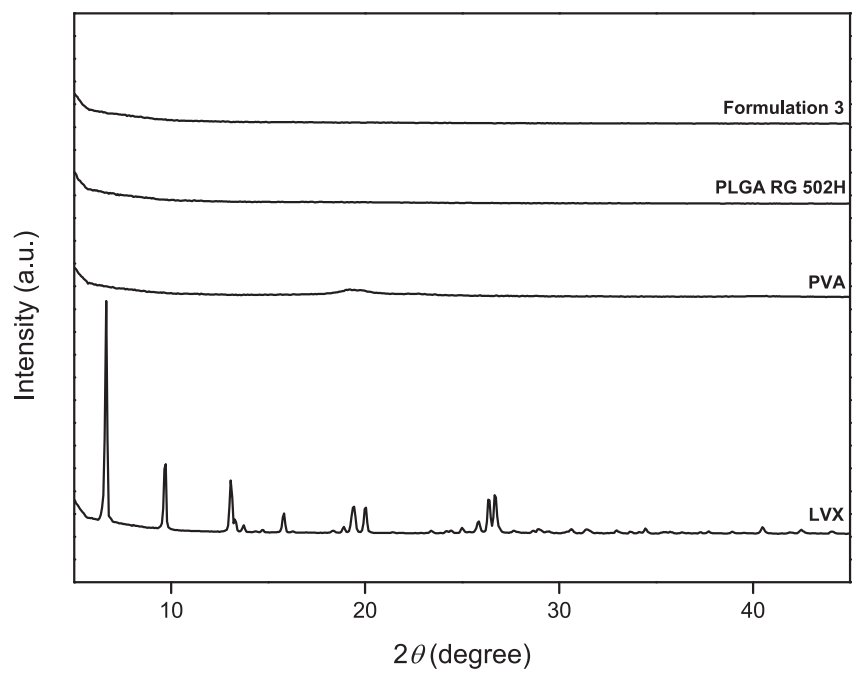

Fig. 4. XRD of LVX, PVA, PLGA RG 502H and the Formulation 3. 
A

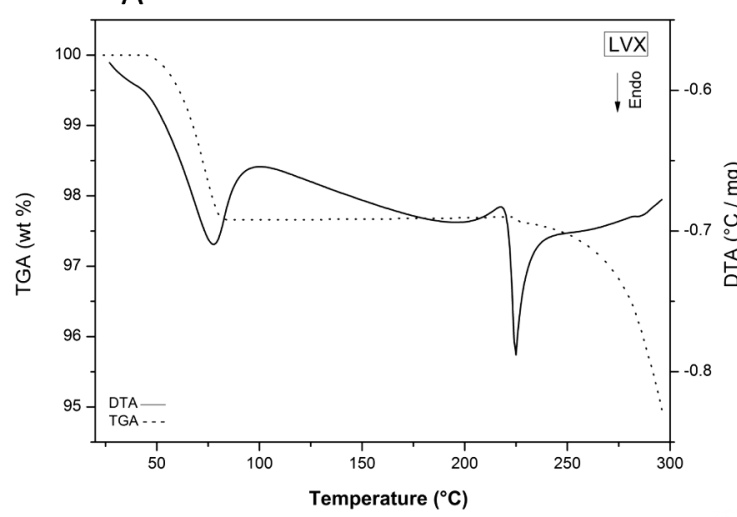

C

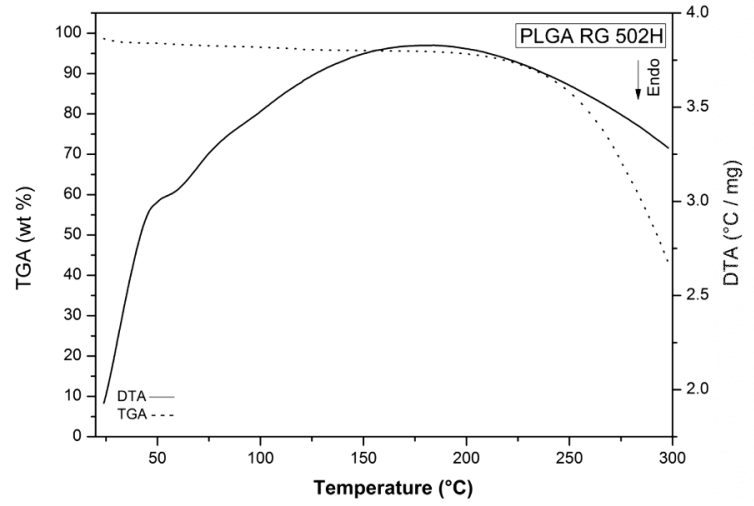

B

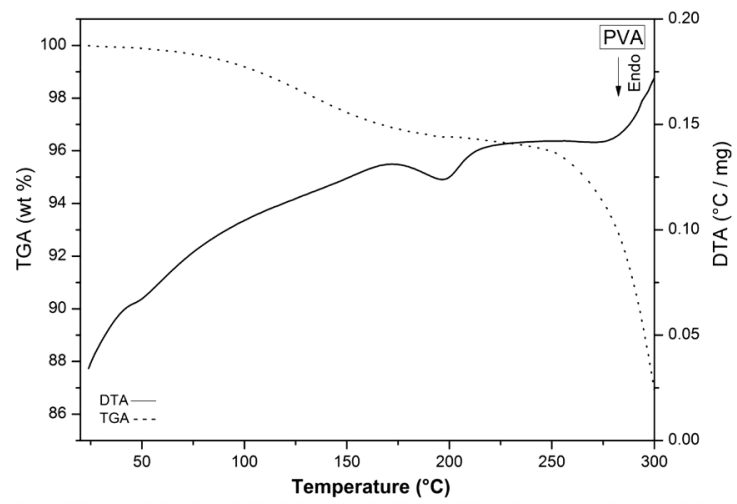

D

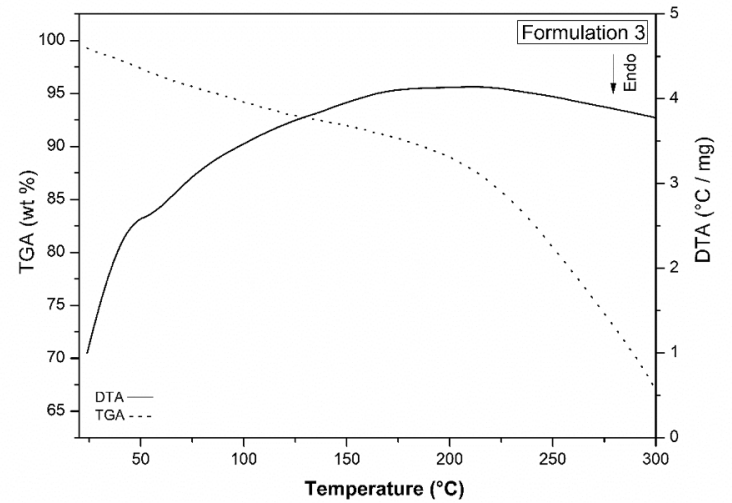

Fig. 5. DTA/TGA thermograms of (A) LVX; (B) PVA; (C) PLGA RG 502H and (D) Formulation 3.

event was attributed to the melting of the anhydrous forms of LVX (Gorman et al., 2012). Experimental conditions do not allow to distinguish the peaks corresponding to the different crystal forms of the anhydrous LVX (Gorman et al., 2012; Kitaoka et al., 1995). The weight loss observed from $240{ }^{\circ} \mathrm{C}$ and above was attributed to LVX degradation (Gorman et al., 2012). For PVA, the melting point was observed close to $200^{\circ} \mathrm{C}$ (Fig. 5B), in agreement with previous work (Sudhamani et al., 2003). The thermograms for the PLGA polymer (Fig. 5C) and the formulation 3 microsphere dry powder (Fig. 5D) are similar: the single event that was observed, i.e. the endothermic transition with an onset temperature close to $50{ }^{\circ} \mathrm{C}$, was attributed to the glass transition temperature of PLGA (Nath et al., 2013). For formulation 3 (Fig. 5D) no peak for the LVX melting was present. Two hypotheses are proposed from the absence of this event: (1) LVX was in an amorphous or dissolved state in the microsphere dry powder or (2) the melting of the PLGA polymer during the analysis resulted in the subsequent dissolution of LVX in the PLGA polymer. None of these hypotheses contradict the conclusions based on the XRD spectrum analysis of the formulation 3 (Fig. 3), i.e. that LVX was solubilized, or dispersed in an amorphous state or as nanocrystals in the PLGA polymer matrix (see 3.2.1.). Noticeably, the percent weight loss at $300{ }^{\circ} \mathrm{C}$ for the formulation 3 was lower than for the PLGA alone, suggesting a higher chemical stability of the microspheres.

\subsubsection{ATR-FTIR}

ATR-FTIR analyses were performed to evaluate the potential chemical alterations or interactions of LVX when incorporated in the MS. Fig. 6 shows the characteristic bands of LVX, PVA, PLGA and LVXloaded PLGA MS (Formulation 3). The LVX spectrum includes bands at 1724, 1618 and $1289 \mathrm{~cm}^{-1}$ corresponding respectively to the $\mathrm{C}=\mathrm{O}$ acid, $\mathrm{C}=\mathrm{O}$ carbonyl and $\mathrm{C}-\mathrm{O}$ acid groups (Gaspar et al., 2015). For PVA, the main observed bands are related to hydroxyl and acetate groups. A large band was obtained at $3300 \mathrm{~cm}^{-1}$ corresponding to $\mathrm{O}-\mathrm{H}$

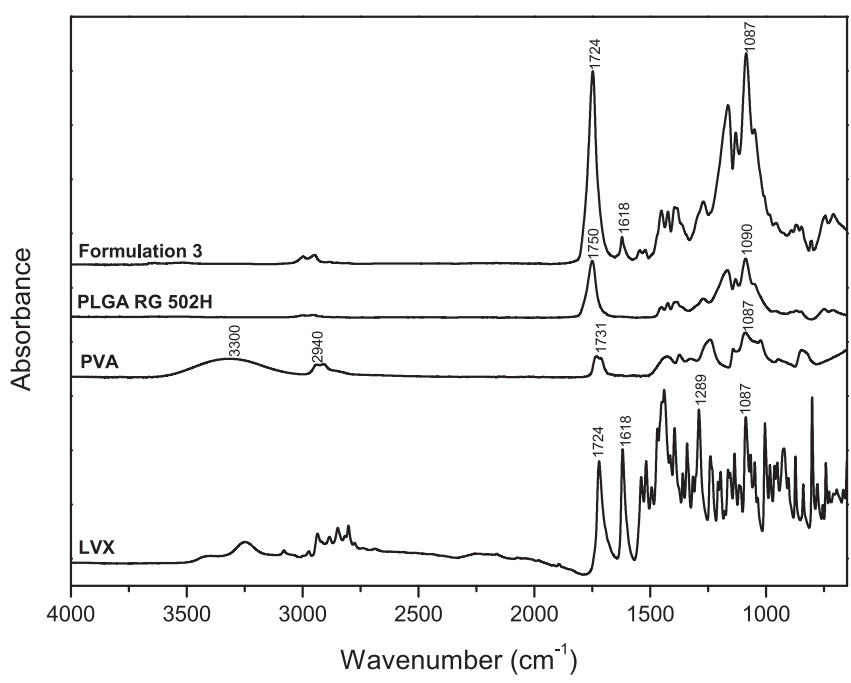

Fig. 6. FTIR spectra of LVX, PVA, PLGA RG 502H and Formulation 3.

stretching. The band present at $2940 \mathrm{~cm}^{-1}$ was attributed to the $\mathrm{C}-\mathrm{H}$ stretching from alkyl groups and at $1731 \mathrm{~cm}^{-1}$ to the stretching from $\mathrm{C}=\mathrm{O}$ and $\mathrm{C}-\mathrm{O}$ from the acetate group (Hema et al., 2009). The PLGA spectrum presented the main peaks at 1750 and $1090 \mathrm{~cm}^{-1}$ corresponding respectively to the $\mathrm{C}=\mathrm{O}$ and $\mathrm{C}-\mathrm{O}$ stretching vibrations (Nath et al., 2013). For the LVX-loaded PLGA MS, the main peaks from LVX were observed, meaning that LVX suffers no alterations when it is incorporated in the polymer matrix.

\subsubsection{Aerodynamic properties}

Using the Handihaler ${ }^{\circledast}$ DPI to aerosolize the powder, the ED value was determined to be $85.0 \pm 3.6 \%$, the FPF was $30.2 \pm 2.3 \%$ and the 

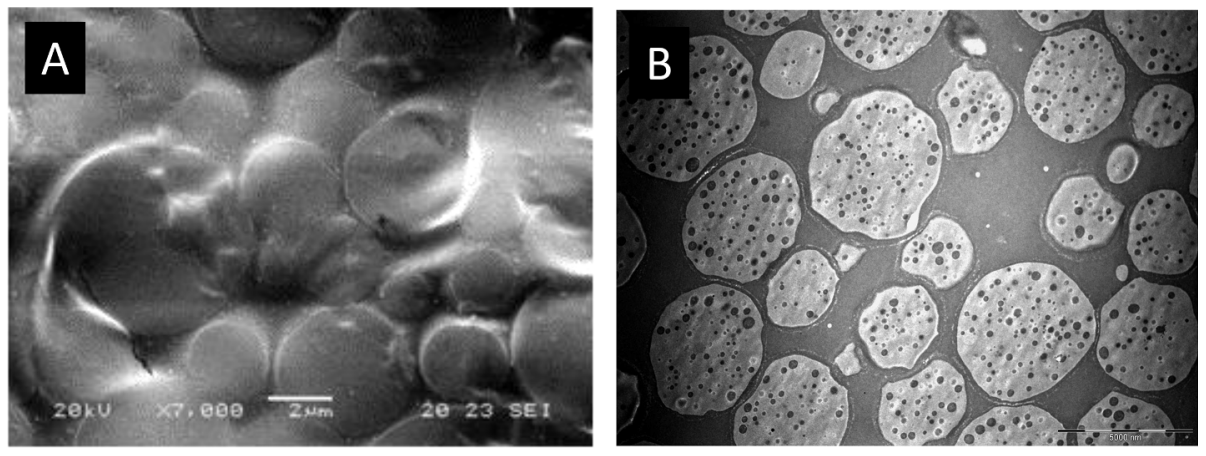

Fig. 7. Cross-sections of $1.5 \mu \mathrm{m}$ and $80 \mathrm{~nm}$ of Formulation 3 respectively observed by SEM (A) and TEM (B).

MMAD $7.1 \pm 0.2 \mu \mathrm{m}$ (mean $\pm \mathrm{SD}$ ). The MMAD was slightly higher than $D_{v}$, fact that can be explained by particle aggregation as observed on the SEM of the dry powder. In spite of the MMAD of $7 \mu \mathrm{m}$, it is still satisfactory for pulmonary administration, and for drug delivery into airways, especially when the particles do not have to reach the alveolar region (Patton and Byron, 2007). Moreover, P. aeruginosa is mainly present in the conductive zone and only colonizes the respiratory zone at advance stages of the disease (Bjarnsholt et al., 2009). Furthermore, as already mentioned, at the obtained particle size, phagocytosis uptake by macrophages is likely to occur. However, and in spite of the few studies that have already studied the dependence of phagocytosis on particle size, maximum values of uptake of polymeric MS by rat alveolar macrophages were reported at a diameter between 2 and $3 \mu \mathrm{m}$ (Champion et al., 2008). Therefore, only a small fraction of the LVXloaded PLGA MS is expected to be phagocytosed in vivo.

\subsubsection{Cross-section analysis}

Cross-sections from the LVX-loaded PLGA MS (Formulation 3) were also analyzed by SEM (Fig. 7A) and TEM (Fig. 7B) in order to inspect the internal morphology of the MS. Both images showed that the MS were filled with polymer and some pores are present. These pores may be responsible for the initial burst effect observed in the in vitro release studies.

\subsubsection{Specific surface area}

BET results from MS (Formulation 3) revealed a surface area of $19.67 \pm 0.315 \mathrm{~m}^{2} / \mathrm{g}$. The surface area was higher than for chitosan MS $\left(6.85 \pm 0.20 \mathrm{~m}^{2} / \mathrm{g}\right)$, already obtained with similar particle size (Gaspar et al., 2015). This is in agreement with the cross-section observations, which indicate the presence of some pores.

\subsubsection{Cytotoxicity study}

The cytotoxicity of PLGA MS prepared according to formulation 3 was evaluated on Calu- 3 cells after $24 \mathrm{~h}$ exposure using the MTS assay (Fig. 8). LVX demonstrated to be relatively well tolerated since it decreased the cell viability close to $50 \%$ only at the highest concentration tested $(1 \mathrm{mg} / \mathrm{mL})$. Values close to $70 \%$ of cell viability were obtained for the concentration of $0.3 \mathrm{mg} / \mathrm{mL}$. This is in agreement with cytotoxicity results already reported for LVX using other epithelial cells from lung bronchus (NL 20 cells and HBE 135 cells). In particular, 60\% cell viability, in the case of NL 20 cells, was observed at a concentration of $0.3 \mathrm{mg} / \mathrm{mL}$ of LVX and for HBE 135 cells, this concentration resulted in $80 \%$ cell survival. Moreover, LVX was shown to be less cytotoxic than other fluoroquinolones, such as moxifloxacin and ciprofloxacin (Dudley et al., 2014). Unloaded PLGA RG 502H MS revealed very low cytotoxicity to the Calu-3 cells. Only at the two highest concentrations (5 and $10 \mathrm{mg} / \mathrm{mL}$ ), cell viability values were lower than $100 \%$, but still close to $90 \%$ (Fig. 8). Similar conclusions have been already made by other authors, with values higher than $90 \%$ of Calu- 3 cell viability for $0.5 \mathrm{mg} / \mathrm{mL}$ of PLGA particles and values close to $80 \%$ for a concentration of $5 \mathrm{mg} / \mathrm{mL}$ (Patel et al., 2012). LVX-loaded PLGA MS

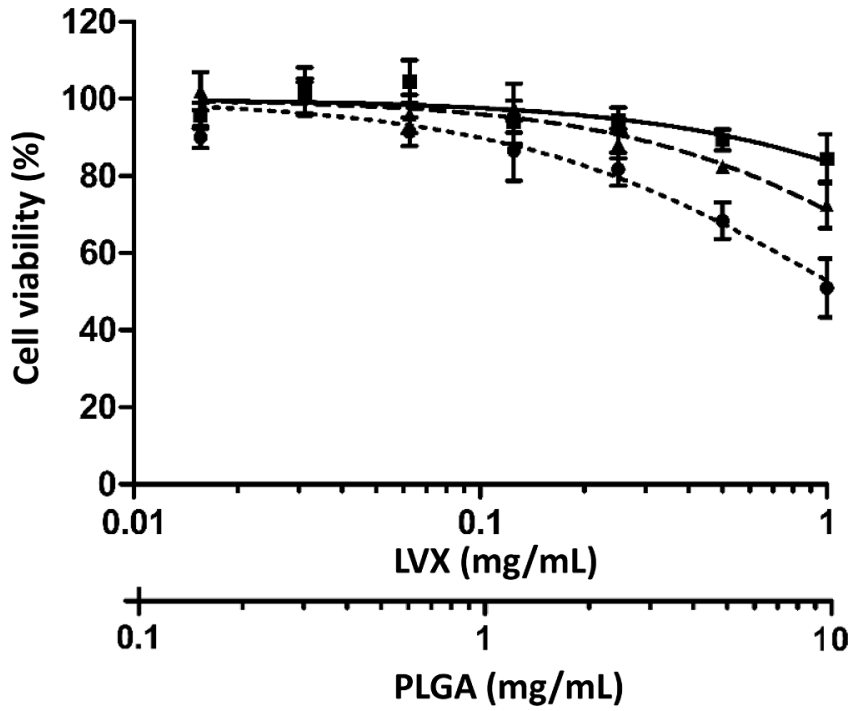

Fig. 8. Calu-3 cell viability determined with MTS assay after $24 \mathrm{~h}$ of incubation with LVX (••-), unloaded MS ( $\bullet-)$ ) and Formulation $3(-\llcorner\cdot)$. Cell viability (percent of control, mean \pm SEM $(n=5)$ ) is expressed as a function of the PLGA concentration and/or LVX concentration.

(Formulation 3) was of intermediate cytotoxicity, (Anderson and Shive, 2012). This finding for the LVX-loaded MS is in agreement with the sustained release properties of the MS. After $24 \mathrm{~h}$ of incubation, values of cell viability close to $70 \%$ (for the highest concentration tested) were obtained. Therefore, around $30 \%$ of the cells died. On the other side, for LVX itself, around $50 \%$ of the cells were dead at the highest concentration tested. Considering zero/ $10 \%$ cytotoxicity from the polymer, this means the formulation 3 showed ca. $60 \%$ of the toxicity of LVX itself. This aligns with the $60-70 \%$ of LVX release from PLGA MS after $24 \mathrm{~h}$ (Fig. 3).

\section{Conclusions}

The incorporation of a drug with high water solubility, such as LVX, in a hydrophobic polymer is of high relevance, but also a challenge in MS preparation. A preliminary method enabled to select the PLGA RG $502 \mathrm{H}$ as the most adequate in terms of drug loading and particle size results. In order to further increase LVX content, some modifications were assessed. A DL close to $10 \%$, suitable mean particle size together with spherical morphology and controlled release properties allowed choosing the Formulation 3, as an interesting system for further analyses. Several techniques demonstrated the good incorporation of LVX in the polymer matrix. The selected formulation also had acceptable aerodynamic diameter and low cytotoxicity with respiratory cells, which are clearly advantageous when compared to LVX itself, by reducing toxicity risk and increasing local concentrations. The present 
PLGA MS-based dry powder is a promising sustained-release formulation for the inhaled delivery of LVX. After its administration, it is expected to achieve high lung concentrations over a prolonged time period, thus reducing the dosing frequency, increasing the patients' compliance, and improving the treatment efficiency.

\section{Conflict of interest}

The authors report no conflicts of interest.

\section{Acknowledgements}

The authors thank FCT (Fundação para a Ciência e a Tecnologia, Portugal) for the financial support through the grant SFRH/BD/80307/ 2011. DTA/TGA, XRD and ATR-FTIR analyses were performed at the University of Poitiers, Pôle Commun de Mesures Physico-chimiques platform. The authors thank Ms. Agnès Audurier, INSERM U 1070 and Faculty of Medicine and Pharmacy, for her technical assistance, and Mr. Christophe Adier, INSERM U 1070 and CHU de Poitiers, for his technical assistance in the spectrophotometric and HPLC determinations and the Quality Management of the analytical process.

\section{References}

Adi, H., Young, P.M., Chan, H.-K., Agus, H., Traini, D., 2010. Co-spray-dried mannitolciprofloxacin dry powder inhaler formulation for cystic fibrosis and chronic obstructive pulmonary disease. Eur. J. Pharm. Sci. 40, 239-247. https://doi.org/10. 1016/j.ejps.2010.03.020.

Anderson, J.M., Shive, M.S., 2012. Biodegradation and biocompatibility of PLA and PLGA microspheres. Adv. Drug Deliv. Rev. 64 (Supplement), 72-82. https://doi.org/10. 1016/j.addr.2012.09.004.

Bjarnsholt, T., Jensen, P.Ø., Fiandaca, M.J., Pedersen, J., Hansen, C.R., Andersen, C.B., Pressler, T., Givskov, M., Høiby, N., 2009. Pseudomonas aeruginosa biofilms in the respiratory tract of cystic fibrosis patients. Pediatr. Pulmonol. 44, 547-558. https:// doi.org/10.1002/ppul.21011.

Champion, J.A., Walker, A., Mitragotri, S., 2008. Role of particle size in phagocytosis of polymeric microspheres. Pharm. Res. 25, 1815-1821. https://doi.org/10.1007/ s11095-008-9562-y.

Diab, R., Brillault, J., Bardy, A., Gontijo, A.V.L., Olivier, J.C., 2012. Formulation and in vitro characterization of inhalable polyvinyl alcohol-free rifampicin-loaded PLGA microspheres prepared with sucrose palmitate as stabilizer: efficiency for ex vivo alveolar macrophage targeting. Int. J. Pharm. 436, 833-839. https://doi.org/10. 1016/j.ijpharm.2012.07.036.

Díez, S., Tros de Ilarduya, C., 2006. Versatility of biodegradable poly(d, 1-lactic-co-glycolic acid) microspheres for plasmid DNA delivery. Eur. J. Pharm. Biopharm. 63, 188-197. https://doi.org/10.1016/j.ejpb.2006.03.007.

Doan, T.V., Couet, W., Olivier, J.C., 2011. Formulation and in vitro characterization of inhalable rifampicin-loaded PLGA microspheres for sustained lung delivery. Int. J. Pharm. 414, 112-117. https://doi.org/10.1016/j.ijpharm.2011.05.007.

Doan, T.V.P., Grégoire, N., Lamarche, I., Gobin, P., Marchand, S., Couet, W., Olivier, J.C., 2013. A preclinical pharmacokinetic modeling approach to the biopharmaceutical characterization of immediate and microsphere-based sustained release pulmonary formulations of rifampicin. Eur. J. Pharm. Sci. 48, 223-230. https://doi.org/10. 1016/j.ejps.2012.10.024.

Doan, T.V.P., Olivier, J.C., 2009. Preparation of rifampicin-loaded PLGA microspheres for lung delivery as aerosol by premix membrane homogenization. Int. J. Pharm. 382, 61-66. https://doi.org/10.1016/j.ijpharm.2009.08.008.

Dudley, M.N., Tsivikovski, R.Y., Griffith, D.C., Rodny, O., 2014. Topical use of levofloxacin for reducing lunf inflammation. In: Mpex Pharmaceuticals, I. (Ed.). Mpex Pharmaceuticals, Inc., Bridgewater, NJ (US), United States.

EMA, 2015. Quinsair - Summary of product characteristics, in: European Medicines Agency, S.M.H. (Ed.). European Medicines Agency, Science Medicines Health.

Emami, J., Hamishehkar, H., Najafabadi, A.R., Gilani, K., Minaiyan, M., Mahdavi, H., Mirzadeh, H., Fakhari, A., Nokhodchi, A., 2009. Particle size design of PLGA microspheres for potential pulmonary drug delivery using response surface methodology. J. Microencapsul. 26, 1-8. https://doi.org/10.1080/02652040802083900.
Food and Drug Administration (FDA), January 2006. USPI Proposed Interactions with Laboratory or Diagnostic Testing.

Foundation, Cystic Fibrosis. 2015. Drug Development Pipeline.

Gaspar, M.C., Couet, W., Olivier, J.C., Pais, A.A.C.C., Sousa, J.J.S., 2013. Pseudomonas aeruginosa infection in cystic fibrosis lung disease and new perspectives of treatment: a review. Eur. J. Clin. Microbiol. Infect. Dis. 32, 1231-1252. https://doi.org/10. 1007/s10096-013-1876-y.

Gaspar, M.C., Grégoire, N., Sousa, J.J.S., Pais, A.A.C.C., Lamarche, I., Gobin, P., Olivier, J.-C., Marchand, S., Couet, W., 2016. Pulmonary pharmacokinetics of levofloxacin in rats after aerosolization of immediate-release chitosan or sustained-release PLGA microspheres. Eur. J. Pharm. Sci. 93, 184-191. https://doi.org/10.1016/j.ejps.2016. 08.024 .

Gaspar, M.C., Sousa, J.J.S., Pais, A.A.C.C., Cardoso, O., Murtinho, D., Serra, M.E.S., Tewes, F., Olivier, J.-C., 2015. Optimization of levofloxacin-loaded crosslinked chitosan microspheres for inhaled aerosol therapy. Eur. J. Pharm. Biopharm. 96, 65-75. https://doi.org/10.1016/j.ejpb.2015.07.010.

Geller, D.E., 2009. Aerosol antibiotics in cystic fibrosis. Respiratory Care 54, 658-670.

Giron Moreno, R.M., Salcedo Posadas, A., Mar Gomez-Punter, R., 2011. Inhaled antibiotic therapy in cystic fibrosis. Archivos de Bronconeumología 47 (Suppl 6), 14-18. https://doi.org/10.1016/S0300-2896(11)70030-8.

Gorman, E.M., Samas, B., Munson, E.J., 2012. Understanding the dehydration of levofloxacin hemihydrate. J. Pharm. Sci. 101, 3319-3330. https://doi.org/10.1002/jps. 23200.

Govender, T., Stolnik, S., Garnett, M.C., Illum, L., Davis, S.S., 1999. PLGA nanoparticles prepared by nanoprecipitation: drug loading and release studies of a water soluble drug. J. Control. Release 57, 171-185. https://doi.org/10.1016/S0168-3659(98) 00116-3.

Guirguis, O.W., Moselhey, M.T.H., 2012. Thermal and structural studies of poly (vinyl alcohol) and hydroxypropyl cellulose blends. Nat. Sci. 4, 11. https://doi.org/10. 4236/ns.2012.41009.

Heijerman, H., Westerman, E., Conway, S., Touw, D., 2009. Inhaled medication and inhalation devices for lung disease in patients with cystic fibrosis: a European consensus. J. Cyst. Fibros. 8, 295-315. https://doi.org/10.1016/j.jcf.2009.04.005.

Hema, M., Selvasekarapandian, S., Arunkumar, D., Sakunthala, A., Nithya, H., 2009. FTIR, XRD and ac impedance spectroscopic study on PVA based polymer electrolyte doped with NH4X (X = Cl, Br, I). J. Non-Cryst. Solids 355, 84-90. https://doi.org/10. 1016/j.jnoncrysol.2008.10.009.

Høiby, N., 2011. Recent advances in the treatment of Pseudomonas aeruginosa infections in cystic fibrosis. BMC Med. 9, 32-38. https://doi.org/10.1186/1741-7015-9-32.

Kitaoka, H., Wada, C., Moroi, R., Hakusui, H., 1995. Effect of dehydration on the formation of levofloxacin pseudopolymorphs. Chem. Pharm. Bull. 43, 649-653. https:// doi.org/10.1248/cpb.43.649.

Kumar, G., Sharma, S., Shafiq, N., Khuller, G.K., Malhotra, S., 2012. Optimization, in vitro-in vivo evaluation, and short-term tolerability of novel levofloxacin-loaded PLGA nanoparticle formulation. J. Pharm. Sci. 101, 2165-2176. https://doi.org/10. 1002/jps. 23087.

Lecaroz, C., Gamazo, C., Renedo, M.J., Blanco-Prieto, M.J., 2006. Biodegradable microand nanoparticles as long-term delivery vehicles for gentamicin. $\mathrm{J}$. Microencapsulation 23, 782-792. https://doi.org/10.1080/02652040600946886.

Nath, S.D., Son, S., Sadiasa, A., Min, Y.K., Lee, B.T., 2013. Preparation and characterization of PLGA microspheres by the electrospraying method for delivering simvastatin for bone regeneration. Int. J. Pharm. 443, 87-94. https://doi.org/10.1016/j. ijpharm.2012.12.037.

Osman, R., Kan, P.L., Awad, G., Mortada, N., El-Shamy, A.-E., Alpar, O., 2013. Spray dried inhalable ciprofloxacin powder with improved aerosolisation and antimicrobial activity. Int. J. Pharm. 449, 44-58. https://doi.org/10.1016/j.ijpharm.2013.04.009.

Patel, B., Gupta, V., Ahsan, F., 2012. PEG-PLGA based large porous particles for pulmonary delivery of a highly soluble drug, low molecular weight heparin. J. Control. Release 162, 310-320. https://doi.org/10.1016/j.jconrel.2012.07.003.

Patton, J.S., Byron, P.R., 2007. Inhaling medicines: delivering drugs to the body through the lungs. Nat. Rev. Drug Discovery 6, 67. https://doi.org/10.1038/nrd2153.

Sah, E., Sah, H., 2015. Recent trends in preparation of poly(lactide-co-glycolide) nanoparticles by mixing polymeric organic solution with antisolvent. J. Nanomater. 2015, 22. https://doi.org/10.1155/2015/794601.

Sudhamani, S.R., Prasad, M.S., Udaya Sankar, K., 2003. DSC and FTIR studies on Gellan and Polyvinyl alcohol (PVA) blend films. Food Hydrocolloids 17, 245-250. https:// doi.org/10.1016/S0268-005X(02)00057-7.

Tewes, F., Paluch, K.J., Tajber, L., Gulati, K., Kalantri, D., Ehrhardt, C., Healy, A.M., 2013. Steroid/mucokinetic hybrid nanoporous microparticles for pulmonary drug delivery. Eur. J. Pharm. Biopharm. 85, 604-613. https://doi.org/10.1016/j.ejpb.2013.03.020.

Tiddens, H.A.W.M., Bos, A.C., Mouton, J.W., Devadason, S., Janssens, H.M., 2014. Inhaled antibiotics: dry or wet? Eur. Respir. J. 44, 1308-1318. https://doi.org/10. 1183/09031936.00090314. 